\title{
Extremal quantum states in coupled systems
}

\author{
K.R. Parthasarathy \\ Indian Statistical Institute, Delhi Centre, 7, S. J. S. Sansanwal Marg, New Delhi-110 016, India
}

Received 2 September 2003; received in revised form 13 October 2003; accepted 22 October 2003

Available online 2 April 2005

In memory of Paul André Meyer

\begin{abstract}
Let $\mathcal{H}_{1}, \mathcal{H}_{2}$ be finite dimensional complex Hilbert spaces describing the states of two finite level quantum systems. Suppose $\rho_{i}$ is a state in $\mathcal{H}_{i}, i=1,2$. Let $\mathcal{C}\left(\rho_{1}, \rho_{2}\right)$ be the convex set of all states $\rho$ in $\mathcal{H}=\mathcal{H}_{1} \otimes \mathcal{H}_{2}$ whose marginal states in $\mathcal{H}_{1}$ and $\mathcal{H}_{2}$ are $\rho_{1}$ and $\rho_{2}$ respectively. Here we present a necessary and sufficient criterion for a $\rho$ in $\mathcal{C}\left(\rho_{1}, \rho_{2}\right)$ to be an extreme point. Such a condition implies, in particular, that for a state $\rho$ to be an extreme point of $\mathcal{C}\left(\rho_{1}, \rho_{2}\right)$ it is necessary that the rank of $\rho$ does not exceed $\left(d_{1}^{2}+d_{2}^{2}-1\right)^{1 / 2}$, where $d_{i}=\operatorname{dim} \mathcal{H}_{i}, i=1,2$. When $\mathcal{H}_{1}$ and $\mathcal{H}_{2}$ coincide with the 1 -qubit Hilbert space $\mathbb{C}^{2}$ with its standard orthonormal basis $\{|0\rangle,|1\rangle\}$ and $\rho_{1}=\rho_{2}=\frac{1}{2} I$ it turns out that a state $\rho \in \mathcal{C}\left(\frac{1}{2} I, \frac{1}{2} I\right)$ is extremal if and only if $\rho$ is of the form $|\Omega\rangle\langle\Omega|$ where $|\Omega\rangle=\frac{1}{\sqrt{2}}\left(|0\rangle\left|\psi_{0}\right\rangle+|1\rangle\left|\psi_{1}\right\rangle\right),\left\{\left|\psi_{0}\right\rangle,\left|\psi_{1}\right\rangle\right\}$ being an arbitrary orthonormal basis of $\mathbb{C}^{2}$. In particular, the extremal states are the maximally entangled states. Using the Weyl commutation relations in the space $L^{2}(A)$ of a finite Abelian group we exhibit a mixed extremal state in $\mathcal{C}\left(\frac{1}{n} I_{n}, \frac{1}{n^{2}} I_{n^{2}}\right)$.

() 2005 Elsevier SAS. All rights reserved.

\section{Résumé}

Soient $\mathcal{H}_{1}$ et $\mathcal{H}_{2}$ des espaces de Hilbert complexes de dimension finies décrivant les états de deux systmes quantiques. Soient $\rho_{1}, \rho_{2}$ deux états sur $\mathcal{H}_{1}$ et $\mathcal{H}_{2}$. Soit $\left(\rho_{1}, \rho_{2}\right)$ le convexe formé par les états sur $\mathcal{H}_{1} \otimes \mathcal{H}_{2}$ induisant $\rho_{1}$ et $\rho_{2}$. L'objet de ce travail est de donner un critère nécessaire et suffisant pour qu'un point $\rho$ de ce convexe soit extrémal. Une condition nécessaire est que le rang de $\rho$ n'excède pas $\left(d_{1}^{2}+d_{2}^{2}-1\right)^{1 / 2}$; ou $d_{i}=\operatorname{dim} \mathcal{H}_{i}$. Lorsque $\mathcal{H}_{1}$ et $\mathcal{H}_{2}$ sont l'espace $\mathbb{C}^{2}$ avec sa base standard $\{|0\rangle|1\rangle\}$ et que $\rho_{1}=\rho_{2}=-I$, les états extrémaux sont caractérisés. Une exemple d'état extrémal mélangé est donné dans $C\left(\frac{1}{n} I_{n}, \frac{1}{n^{2}} I_{n^{2}}\right)$.

(c) 2005 Elsevier SAS. All rights reserved.
\end{abstract}

Keywords: Coupled quantum systems; Marginal states; Extreme points; Doubly stochastic matrices; Separable and nonseparable states

E-mail address: krp@isid.ac.in (K.R. Parthasarathy). 


\section{Introduction}

One of the well-known problems of classical probability theory is the determination of the set of all extreme points in the convex set of all probability distributions in a product Borel space $(X \times Y, \mathcal{F} \times \mathcal{G})$ with fixed marginal distributions $\mu$ and $v$ on $(X, \mathcal{F})$ and $(Y, \mathcal{G})$ respectively. Denote this convex set by $C(\mu, v)$. When $X=Y=$ $\{1,2, \ldots, n\}, \mathcal{F}=\mathcal{G}$ is the field of all subsets of $X$ and $\mu=v$ is the uniform distribution then the problem is answered by the famous theorem of Birkhoff and von Neumann [1,2] that the set of extreme points of the convex set of all doubly stochastic matrices of order $n$ is the set of all permutation matrices of order $n$. Problems of this kind have a natural analogue in quantum probability. Suppose $\mathcal{H}_{1}$ and $\mathcal{H}_{2}$ are finite dimensional complex Hilbert spaces describing the states of two finite level quantum systems $S_{1}$ and $S_{2}$ respectively. Then the Hilbert space of the coupled system $S_{12}$ is $\mathcal{H}_{1} \otimes \mathcal{H}_{2}$. Suppose $\rho_{i}$ is a state of $S_{i}$ in $\mathcal{H}_{i}, i=1,2$. Any state $\rho$ in $S_{12}$ yields marginal states $\operatorname{Tr}_{\mathcal{H}_{2}} \rho$ in $\mathcal{H}_{1}$ and $\operatorname{Tr}_{\mathcal{H}_{1}} \rho$ in $\mathcal{H}_{2}$ where $\operatorname{Tr}_{\mathcal{H}_{i}}$ is the relative trace over $\mathcal{H}_{i}$. Denote by $\mathcal{C}\left(\rho_{1}, \rho_{2}\right)$ the convex set of all states $\rho$ of the coupled system $S_{12}$ whose marginal states in $\mathcal{H}_{1}$ and $\mathcal{H}_{2}$ are $\rho_{1}$ and $\rho_{2}$ respectively. One would like to have a complete description of the set of all extreme points of $\mathcal{C}\left(\rho_{1}, \rho_{2}\right)$. In this paper we shall present a necessary and sufficient criterion for an element $\rho$ in $\mathcal{C}\left(\rho_{1}, \rho_{2}\right)$ to be an extreme point. This leads to an interesting (and perhaps surprising) upper bound on the rank of such an extremal state $\rho$. Indeed, if $\rho$ is an extreme point of $\mathcal{C}\left(\rho_{1}, \rho_{2}\right)$ then the rank of $\rho$ cannot exceed $\left(d_{1}^{2}+d_{2}^{2}-1\right)^{1 / 2}$ where $d_{i}=\operatorname{dim} \mathcal{H}_{i}$. Note that the rank of an arbitrary state in $\mathcal{H}_{1} \otimes \mathcal{H}_{2}$ can vary from 1 to $d_{1} d_{2}$. When $\mathcal{H}_{1}=\mathcal{H}_{2}=\mathbb{C}^{2},\{|0\rangle,|1\rangle\}$ is the standard (computational) basis of $\mathbb{C}^{2}$ and $\rho_{1}=\rho_{2}=\frac{1}{2} I$ it turns out that a state $\rho$ in $\mathcal{C}\left(\frac{1}{2} I, \frac{1}{2} I\right)$ is extremal if and only if $\rho$ has the form $|\Omega\rangle\langle\Omega|$ where $|\Omega\rangle=\frac{1}{\sqrt{2}}\left(|0\rangle\left|\psi_{0}\right\rangle+|1\rangle\left|\psi_{1}\right\rangle\right),\left\{\left|\psi_{0}\right\rangle,\left|\psi_{1}\right\rangle\right\}$ being any orthonormal basis of $\mathbb{C}^{2}$. These are the well-known maximally entangled states.

A major part of this work was done by the author during his visit to the University of Greifswald during 17 June16 July under a DST (India)-DAAD (Germany) project between the Indian Statistical Institute and the mathematics department of the University of Greifswald. The author is grateful to these organisations for their generous support. The hospitality extended by the colleagues of the Quantum Probability group in the University of Greifswald and, particularly, Michael Schurmann is gratefully acknowledged. The example of Section 4 was constructed during the author's visit to the University of Nottingham during 13-25 August, 2003 when he enjoyed the hospitality of R.L. Hudson and J.M. Lindsay. Finally, the author expresses his thanks to Mr. Anil Shukla for his help in texing the manuscript.

\section{Extreme points of the convex set $\mathcal{C}\left(\rho_{1}, \rho_{2}\right)$}

In the analysis of extreme points in a compact convex set of positive definite matrices the following proposition plays an important role [7]. See also [3,4] and [6].

Proposition 2.1. Let $\rho$ be any positive definite matrix of order $n$ and $\operatorname{rank} k<n$. Then there exists a permutation matrix $\sigma$ of order $n, a k \times(n-k)$ matrix $A$ and a strictly positive definite matrix $K$ of order $k$ such that

$$
\sigma \rho \sigma^{-1}=\left[\begin{array}{c|c}
K & K A \\
\hline A^{\dagger} K & A^{\dagger} K A
\end{array}\right] .
$$

If, in addition, $\rho=\frac{1}{2}\left(\rho^{\prime}+\rho^{\prime \prime}\right)$ where $\rho^{\prime}$ and $\rho^{\prime \prime}$ are also positive definite matrices then there exist positive definite matrices $K^{\prime}, K^{\prime \prime}$ of order $k$ such that

$$
\sigma \rho^{\#} \sigma^{-1}=\left[\begin{array}{c|c}
K^{\#} & K^{\#} A \\
\hline A^{\dagger} K^{\#} & A^{\dagger} K^{\#} A
\end{array}\right],
$$

where \# indicates 1 and $\%$. 
Proof. Choose vectors $\boldsymbol{u}_{i} \in \mathbb{C}^{n}, i=1,2, \ldots, n$, such that

$$
\rho=\left(\left(\left\langle\boldsymbol{u}_{i} \mid \boldsymbol{u}_{j}\right\rangle\right)\right), \quad i, j \in\{1,2, \ldots, n\} .
$$

Since rank $\rho=k$, the linear span of all the $\boldsymbol{u}_{i}$ 's has dimension $k$. Hence modulo a permutation $\sigma$ of $\{1,2, \ldots, n\}$ we may assume that $\boldsymbol{u}_{1}, \boldsymbol{u}_{2}, \ldots, \boldsymbol{u}_{k}$ are linearly independent and

$$
\boldsymbol{u}_{k+j}=a_{1 j} \boldsymbol{u}_{1}+a_{2 j} \boldsymbol{u}_{2}+\cdots+a_{k j} \boldsymbol{u}_{k}, \quad 1 \leqslant j \leqslant n-k .
$$

Putting

$$
\begin{aligned}
& K=\left(\left(\left\langle\boldsymbol{u}_{i} \mid \boldsymbol{u}_{j}\right\rangle\right)\right), \quad i, j \in 1,2, \ldots, k, \\
& A=\left(\left(a_{i j}\right)\right), \quad i=1,2, \ldots, k ; j=1,2, \ldots, n-k,
\end{aligned}
$$

and denoting by the same letter $\sigma$, the permutation unitary matrix of order $n$ corresponding to $\sigma$ we obtain the relation (2.1). To prove the second part we express

$$
\sigma \rho \sigma^{-1}=\left[\begin{array}{c|c}
K & K A \\
\hline A^{\dagger} K & A^{\dagger} K A
\end{array}\right]=\frac{1}{2}\left[\begin{array}{c|c}
K^{\prime} & B_{1} \\
\hline B_{1}^{\dagger} & C_{1}
\end{array}\right]+\frac{1}{2}\left[\begin{array}{c|c}
K^{\prime \prime} & B_{2} \\
\hline B_{2}^{\dagger} & C_{2}
\end{array}\right]
$$

where the two partitioned matrices on the right-hand side are the matrices $\sigma \rho^{\prime} \sigma^{-1}$ and $\sigma \rho^{\prime \prime} \sigma^{-1}$. Now construct vectors $\boldsymbol{v}_{i}, \boldsymbol{w}_{i}, i=1,2, \ldots, n$, such that

$$
\begin{aligned}
& \sigma \rho^{\prime} \sigma^{-1}=\left(\left(\left\langle\boldsymbol{v}_{i} \mid \boldsymbol{v}_{j}\right\rangle\right)\right), \quad i, j \in\{1,2, \ldots, n\}, \\
& \sigma \rho^{\prime \prime} \sigma^{-1}=\left(\left(\left\langle\boldsymbol{w}_{i} \mid \boldsymbol{w}_{j}\right\rangle\right)\right), \quad i, j \in\{1,2, \ldots, n\} .
\end{aligned}
$$

Let $|0\rangle,|1\rangle$ be the standard orthonormal basis of $\mathbb{C}^{2}$. Define

$$
\left|\boldsymbol{\varphi}_{i}\right\rangle=\frac{1}{\sqrt{2}}\left(\left|\boldsymbol{v}_{i}\right\rangle|0\rangle+\left|\boldsymbol{w}_{i}\right\rangle|1\rangle\right), \quad 1 \leqslant i \leqslant n .
$$

Then we have

$$
\left\langle\boldsymbol{\varphi}_{i} \mid \boldsymbol{\varphi}_{j}\right\rangle=\frac{1}{2}\left(\left\langle\boldsymbol{v}_{i} \mid \boldsymbol{v}_{j}\right\rangle+\left\langle\boldsymbol{w}_{i} \mid \boldsymbol{w}_{j}\right\rangle\right)=\left\langle\boldsymbol{u}_{i} \mid \boldsymbol{u}_{j}\right\rangle \quad \text { for all } i, j \in\{1,2, \ldots, n\} .
$$

Thus the correspondence $\boldsymbol{u}_{i} \rightarrow \boldsymbol{\varphi}_{i}$ is an isometry. Hence by (2.3) we have

$$
\boldsymbol{\varphi}_{k+j}=a_{1 j} \boldsymbol{\varphi}_{1}+a_{2 j} \boldsymbol{\varphi}_{2}+\cdots+a_{k j} \boldsymbol{\varphi}_{k}, \quad 1 \leqslant j \leqslant n-k .
$$

Substituting for the $\varphi_{i}$ 's from (2.6) and using the orthogonality of $|0\rangle$ and $|1\rangle$ we conclude that

$$
\begin{aligned}
& \left|\boldsymbol{v}_{k+j}\right\rangle=\sum_{i=1}^{k} a_{i j}\left|\boldsymbol{v}_{i}\right\rangle, \\
& \left|\boldsymbol{w}_{k+j}\right\rangle=\sum_{i=1}^{k} a_{i j}\left|\boldsymbol{w}_{i}\right\rangle .
\end{aligned}
$$

Putting

$$
\begin{aligned}
& K^{\prime}=\left(\left(\left\langle\boldsymbol{v}_{i} \mid \boldsymbol{v}_{j}\right\rangle\right)\right), \quad i, j \in\{1,2, \ldots, k\}, \\
& K^{\prime \prime}=\left(\left(\left\langle\boldsymbol{w}_{i} \mid \boldsymbol{w}_{j}\right\rangle\right)\right), \quad i, j \in=\{1,2, \ldots, k\},
\end{aligned}
$$

and substituting (2.7) and (2.8) in (2.4) and (2.5) we obtain $B_{1}=K^{\prime} A, C_{1}=A^{\dagger} K^{\prime} A, B_{2}=K^{\prime \prime} A, C_{2}=A^{\dagger} K^{\prime \prime} A$. Thus we have (2.2). 
Let $\mathcal{H}_{1}, \mathcal{H}_{2}$ be two complex Hilbert spaces of finite dimension $d_{1}, d_{2}$ and equipped with orthonormal bases $\left\{\boldsymbol{e}_{1}, \boldsymbol{e}_{2}, \ldots, \boldsymbol{e}_{d_{1}}\right\},\left\{\boldsymbol{f}_{1}, \boldsymbol{f}_{2}, \ldots, \boldsymbol{f}_{d_{2}}\right\}$ respectively. Consider the tensor product $\mathcal{H}=\mathcal{H}_{1} \otimes \mathcal{H}_{2}$ equipped with the orthonormal basis $\boldsymbol{g}_{i j}=\boldsymbol{e}_{i} \otimes \boldsymbol{f}_{j}$ with the ordered pairs $i j$ in the lexicographic order. For any operator $X$ on $\mathcal{H}$ we associate its marginal operators $X_{i}$ in $\mathcal{H}_{i}$ by putting

$$
X_{1}=\operatorname{Tr}_{\mathcal{H}_{2}} X, \quad X_{2}=\operatorname{Tr}_{\mathcal{H}_{1}} X
$$

where $\operatorname{Tr}_{\mathcal{H}_{i}}$ stands for the relative trace over $\mathcal{H}_{i}$. If $\rho$ is a state on $\mathcal{H}$, i.e., a positive operator of unit trace, then its marginal operators are states in $\mathcal{H}_{1}$ and $\mathcal{H}_{2}$. Now we fix two states $\rho_{1}$ and $\rho_{2}$ in $\mathcal{H}_{1}$ and $\mathcal{H}_{2}$ respectively and consider the compact convex set

$$
\mathcal{C}\left(\rho_{1}, \rho_{2}\right)=\left\{\rho \mid \rho \text { a state on } \mathcal{H} \text { with marginals } \rho_{1} \text { and } \rho_{2} \text { in } \mathcal{H}_{1} \text { and } \mathcal{H}_{2} \text { respectively }\right\}
$$

in $\mathcal{B}(\mathcal{H})$. Let $\mathcal{E}\left(\rho_{1}, \rho_{2}\right) \subset \mathcal{C}\left(\rho_{1}, \rho_{2}\right)$ be the set of all extreme points in $\mathcal{C}\left(\rho_{1}, \rho_{2}\right)$.

Proposition 2.2. Let $\rho \in \mathcal{E}\left(\rho_{1}, \rho_{2}\right)$. Then $\rho$ is singular.

Proof. Suppose $\rho$ is nonsingular. Choose nonzero Hermitian operators $L_{i}$ in $\mathcal{H}_{i}$ with zero trace. Then for all sufficiently small and positive $\varepsilon$, the operators $\rho \pm \varepsilon L_{1} \otimes L_{2}$ are positive definite. Since the marginal operators of $L_{1} \otimes L_{2}$ are 0 , both of the operators $\rho \pm \varepsilon L_{1} \otimes L_{2}$ belong to $\mathcal{C}\left(\rho_{1}, \rho_{2}\right)$ and

$$
\rho=\frac{1}{2}\left(\left(\rho+\varepsilon L_{1} \otimes L_{2}\right)+\left(\rho-\varepsilon L_{1} \otimes L_{2}\right)\right)
$$

and $\rho$ is not extremal.

Proposition 2.3. Let $n=d_{1} d_{2}, \rho \in \mathcal{C}\left(\rho_{1}, \rho_{2}\right), \operatorname{rank} \rho=k<n$ and let $\sigma$ be a permutation of the ordered basis $\left\{\boldsymbol{g}_{i j}\right\}$ of $\mathcal{H}$ such that

$$
\sigma \rho \sigma^{-1}=\left[\begin{array}{c|c}
K & K A \\
\hline A^{\dagger} K & A^{\dagger} K A
\end{array}\right],
$$

where $K$ is a strictly positive definite matrix of order $k$. Then, in order that $\rho \in \mathcal{E}\left(\rho_{1}, \rho_{2}\right)$ it is necessary that there exists no nonzero Hermitian matrix $L$ of order $k$ such that both the marginal operators of

$$
\sigma^{-1}\left[\begin{array}{c|c}
L & L A \\
\hline A^{\dagger} L & A^{\dagger} L A
\end{array}\right] \sigma
$$

vanish.

Proof. Suppose there exists a nonzero Hermitian matrix $L$ of order $k$ such that both the marginals of the operator (2.10) vanish. Since $K$ in (2.9) is nonsingular and positive definite it follows that for all sufficiently small and positive $\varepsilon$, the matrices $K \pm \varepsilon L$ are strictly positive definite. Hence

$$
\rho=\frac{1}{2}\left\{\sigma^{-1}\left[\begin{array}{c|c}
K+\varepsilon L & (K+\varepsilon L) A \\
\hline A^{\dagger}(K+\varepsilon L) & A^{\dagger}(K+\varepsilon L) A
\end{array}\right] \sigma+\sigma^{-1}\left[\begin{array}{c|c}
K-\varepsilon L & (K-\varepsilon L) A \\
\hline A^{\dagger}(K-\varepsilon L) & A^{\dagger}(K-\varepsilon L) A
\end{array}\right] \sigma\right\}
$$

where each summand on the right-hand side has the same marginal operators as $\rho$. Furthermore

$$
\left[\begin{array}{c|c}
K \pm \varepsilon L & (K \pm \varepsilon L) \\
\hline A^{\dagger}(K \pm \varepsilon L) & A^{\dagger}(K \pm \varepsilon L) A
\end{array}\right]=\left[\frac{I}{A^{\dagger}}\right](K \pm \varepsilon L)[I \mid A] \geqslant 0 .
$$

Thus $\rho$ is not extremal. 
Corollary. Let $\rho \in \mathcal{E}\left(\rho_{1}, \rho_{2}\right)$. Then rank $\rho \leqslant \sqrt{d_{1}^{2}+d_{2}^{2}-1}$.

Proof. Let $\operatorname{rank} \rho=k$. By Proposition 2.2, $k<n$. Since $\rho$ is a positive definite matrix in the basis $\left\{\boldsymbol{g}_{i j}\right\}$ such that $\sigma \rho \sigma^{-1}$ can be expressed in the form (2.9). The extremality of $\rho$ implies that there exists no nonzero Hermitian matrix $L$ of order $k$ such that the matrix (2.10) has both its marginals equal to 0 . The vanishing of both the marginals of (2.10) is equivalent to

$$
\operatorname{Tr} \sigma^{-1}\left[\begin{array}{c|c}
L & L A \\
\hline A^{\dagger} L & A^{\dagger} L A
\end{array}\right] \sigma\left(X_{1} \otimes I^{(2)}+I^{(1)} \otimes X_{2}\right)=0
$$

for all Hermitian operators $X_{i}$ in $\mathcal{H}_{i}, I^{(i)}$ being the identity operator in $\mathcal{H}_{i}$. Eq. (2.11) can be expressed as

$$
\operatorname{Tr} L\left[I_{k} \mid A\right] \sigma\left(X_{1} \otimes I^{(2)}+I^{(1)} \otimes X_{2}\right) \sigma^{-1}\left[\frac{I_{k}}{A^{\dagger}}\right]=0 .
$$

In other words $L$ is in the orthogonal complement of the real linear space

$$
\mathcal{D}=\left\{\left[I_{k} \mid A\right] \sigma\left(X_{1} \otimes I^{(2)}+I^{(1)} \otimes X_{2}\right) \sigma^{-1}\left[\frac{I_{k}}{A^{\dagger}}\right] \mid X_{i} \text { Hermitian in } \mathcal{H}_{i}, i=1,2\right\},
$$

with respect to the scalar product $\langle L \mid M\rangle=\operatorname{Tr} L M$ between any two Hermitian matrices of order $k$. Thus the extremality of $\rho$ implies that $\mathcal{D}^{\perp}=\{0\}$. The real linear space of all Hermitian matrices of order $k$ has dimension $k^{2}$. The real linear space of all Hermitian operators of the form $X_{1} \otimes I^{(2)}+I^{(1)} \otimes X_{2}$ is $d_{1}^{2}+d_{2}^{2}-1$. Thus $k^{2}=\operatorname{dim} \mathcal{D} \leqslant$ $d_{1}^{2}+d_{2}^{2}-1$.

Proposition 2.4. Let $\rho \in \mathcal{C}\left(\rho_{1}, \rho_{2}\right), k, \sigma, K, A$ be as in Proposition 2.3. Suppose there is no nonzero Hermitian matrix $L$ of order $k$ such that both the marginal operators of

$$
\sigma^{-1}\left[\begin{array}{c|c}
L & L A \\
\hline A^{\dagger} L & A^{\dagger} L A
\end{array}\right] \sigma
$$

vanish. Then $\rho \in \mathcal{E}\left(\rho_{1}, \rho_{2}\right)$.

Proof. Suppose $\rho \notin \mathcal{E}\left(\rho_{1}, \rho_{2}\right)$. Then there exist two distinct states $\rho^{\prime}, \rho^{\prime \prime}$ in $\mathcal{C}\left(\rho_{1}, \rho_{2}\right)$ such that

$$
\rho=\frac{1}{2}\left(\rho^{\prime}+\rho^{\prime \prime}\right), \quad \rho^{\prime} \neq \rho^{\prime \prime} .
$$

Since rank $\rho=k$ it follows from Proposition 2.1 that there exist positive definite matrices $K^{\prime}, K^{\prime \prime}$ of order $k$ such that

$$
\sigma \rho^{\#} \sigma^{-1}=\left[\begin{array}{c|c}
K^{\#} & K^{\#} A \\
\hline A^{\dagger} K^{\#} & A^{\dagger} K^{\#} A
\end{array}\right]
$$

where $\left(\rho^{\#}, K^{\#}\right)$ stands for any of the three pairs $(\rho, K),\left(\rho^{\prime}, K^{\prime}\right),\left(\rho^{\prime \prime}, K^{\prime \prime}\right)$. Since $\rho^{\prime} \neq \rho^{\prime \prime}$ and hence $\sigma \rho^{\prime} \sigma^{-1} \neq$ $\sigma \rho^{\prime \prime} \sigma^{-1}$ it follows that $K^{\prime} \neq K^{\prime \prime}$. Putting $L=K^{\prime}-K^{\prime \prime} \neq 0$ we obtain a nonzero Hermitian matrix $L$ of order $k$ such that both the marginal operators of

$$
\sigma^{-1}\left[\begin{array}{c|c}
L & L A \\
\hline A^{\dagger} L & A^{\dagger} L A
\end{array}\right] \sigma
$$

vanish. This is a contradiction.

Combining Proposition 2.3, its corollary and Proposition 2.4 we have the following theorem. 
Theorem 2.5. Let $\mathcal{H}_{1}, \mathcal{H}_{2}$ be complex finite dimensional Hilbert spaces of dimension $d_{1}, d_{2}$ respectively. Suppose $\mathcal{C}\left(\rho_{1}, \rho_{2}\right)$ is the convex set of all states $\rho$ in $\mathcal{H}=\mathcal{H}_{1} \otimes \mathcal{H}_{2}$ whose marginal states in $\mathcal{H}_{1}$ and $\mathcal{H}_{2}$ are $\rho_{1}$ and $\rho_{2}$ respectively. Let $\left\{\boldsymbol{e}_{i}\right\},\left\{\boldsymbol{f}_{j}\right\}$ be orthonormal bases for $\mathcal{H}_{1}, \mathcal{H}_{2}$ respectively and let $\boldsymbol{g}_{i j}=\boldsymbol{e}_{i} \otimes \boldsymbol{f}_{j}, i=1,2, \ldots, d_{1}$; $j=1,2, \ldots, d_{2}$ be the orthonormal basis of $\mathcal{H}$ in the lexicographic ordering of the ordered pairs $i j$. In order that an element $\rho$ in $\mathcal{C}\left(\rho_{1}, \rho_{2}\right)$ be an extreme point it is necessary that its $\operatorname{rank} k$ does not exceed $\sqrt{d_{1}^{2}+d_{2}^{2}-1}$. Let $\sigma$ be a permutation unitary operator in $\mathcal{H}$, permuting the basis $\left\{\boldsymbol{g}_{i j}\right\}$ and satisfying

$$
\sigma \rho \sigma^{-1}=\left[\begin{array}{c|c}
K & K A \\
\hline A^{\dagger} K & A^{\dagger} K A
\end{array}\right]
$$

where $K$ is a strictly positive definite matrix of order $k$. Then $\rho$ is an extreme point of the convex set $\mathcal{C}\left(\rho_{1}, \rho_{2}\right)$ if and only if the real linear space

$$
\mathcal{D}=\left\{\left[I_{k} \mid A\right] \sigma\left(X_{1} \otimes I^{(2)}+I^{(1)} \otimes X_{2}\right) \sigma^{-1}\left[\frac{I}{A^{\dagger}}\right] \mid X_{i} \text { Hermitian in } \mathcal{H}_{i}, i=1,2\right\}
$$

coincides with the space of all Hermitian matrices of order $k$.

Proof. Immediate from Proposition 2.3, its corollary and Proposition 2.4.

\section{The case $\mathcal{H}_{1}=\mathcal{H}_{2}=\mathbb{C}^{2}$}

We consider the orthonormal basis

$$
|0\rangle=\left[\begin{array}{l}
1 \\
0
\end{array}\right], \quad|1\rangle=\left[\begin{array}{l}
0 \\
1
\end{array}\right]
$$

in $\mathbb{C}^{2}$ and write

$$
|x y\rangle=|x\rangle \otimes|y\rangle \quad \text { for all } x, y \in\{0,1\} .
$$

Then $\boldsymbol{e}_{1}=|00\rangle, \boldsymbol{e}_{2}=|01\rangle, \boldsymbol{e}_{3}=|10\rangle, \boldsymbol{e}_{4}=|11\rangle$ constitute an ordered orthonormal basis for $\mathbb{C}^{2} \otimes \mathbb{C}^{2}$. For any state $\rho$ in $\mathbb{C}^{2} \otimes \mathbb{C}^{2}$ define

$$
K_{\rho}\left((x, y),\left(x^{\prime}, y^{\prime}\right)\right)=\left\langle x y|\rho| x^{\prime} y^{\prime}\right\rangle, \quad x, y, x^{\prime}, y^{\prime} \in\{0,1\} .
$$

If $\rho$ has marginal states $\rho_{1}, \rho_{2}$ then

$$
\begin{aligned}
& K_{\rho}\left((x, 0),\left(x^{\prime}, 0\right)\right)+K_{\rho}\left((x, 1),\left(x^{\prime}, 1\right)\right)=\left\langle x\left|\rho_{1}\right| x^{\prime}\right\rangle, \\
& K_{\rho}\left((0, y),\left(0, y^{\prime}\right)\right)+K_{\rho}\left((1, y),\left(1, y^{\prime}\right)\right)=\left\langle y\left|\rho_{2}\right| y^{\prime}\right\rangle
\end{aligned}
$$

for all $x, y, x^{\prime}, y^{\prime}$ in $\{0,1\}$. If $\rho$ is an extreme point of the convex set $\mathcal{C}\left(\rho_{1}, \rho_{2}\right)$ it follows from Theorem 2.5 that the rank of $\rho$ cannot exceed $\sqrt{7}$. In other words, every extremal state $\rho^{\prime}$ in $\mathcal{C}\left(\rho_{1}, \rho_{2}\right)$ has rank 1 or 2 . When $\rho_{1}=\rho_{2}=\frac{1}{2} I$ we have the following theorem:

Theorem 3.1. Let $\mathcal{H}_{1}=\mathcal{H}_{2}=\mathbb{C}^{2}$. A state $\rho$ in $\mathcal{C}\left(\frac{1}{2} I, \frac{1}{2} I\right)$ is an extreme point if and only if $\rho=|\Omega\rangle\langle\Omega|$ where

$$
|\Omega\rangle=\frac{1}{\sqrt{2}}\left(|0\rangle \otimes\left|\psi_{0}\right\rangle+|1\rangle \otimes\left|\psi_{1}\right\rangle\right)
$$

$\left\{\left|\psi_{0}\right\rangle,\left|\psi_{1}\right\rangle\right\}$ being an orthonormal basis of $\mathbb{C}^{2}$. 
Proof. We shall first show that there is no extremal state $\rho$ of rank 2 in $\mathcal{C}\left(\frac{1}{2} I, \frac{1}{2} I\right)$. To this end choose and fix a state $\rho$ of rank 2 in $\mathcal{C}\left(\frac{1}{2} I, \frac{1}{2} I\right)$. Then the right-hand sides of (3.2) and (3.3) coincide with $\frac{1}{2} \delta_{x x^{\prime}}$ and $\frac{1}{2} \delta_{y y^{\prime}}$ respectively and in the ordered basis $\left\{\boldsymbol{e}_{j}, 1 \leqslant j \leqslant 4\right\}$ the positive definite matrix $K_{\rho}$ of rank 2 in (3.1) assumes the form

$$
K_{\rho}=\left[\begin{array}{cccc}
\frac{a}{2} & x & y & z \\
\bar{x} & \frac{1-a}{2} & t & -y \\
\bar{y} & \bar{t} & \frac{1-a}{2} & -x \\
\bar{z} & -\bar{y} & -\bar{x} & \frac{a}{2}
\end{array}\right]
$$

for some $0 \leqslant a \leqslant 1, x, y, z, t \in \mathbb{C}$. The fact $K_{\rho}$ has rank 2 implies that one of the following three cases holds:

(1) $\left[\begin{array}{cc}a / 2 & x \\ \bar{x} & (1-a) / 2\end{array}\right]$ is strictly positive definite;

(2) $\left[\begin{array}{cc}a / 2 & y \\ \bar{y} & (1-a) / 2\end{array}\right]$ is strictly positive definite;

(3) $|x|^{2}=|y|^{2}=\frac{a(1-a)}{4}$ and one of the matrices $\left[\begin{array}{cc}a / 2 & z \\ \bar{z} & a / 2\end{array}\right],\left[\begin{array}{cc}(1-a) / 2 & t \\ & (1-a) / 2\end{array}\right]$ is strictly positive definite.

We shall first show that case (3) is vacuous. We assume that

$$
|x|^{2}=|y|^{2}=\frac{a(1-a)}{4}, \quad|z|^{2}<\frac{a^{2}}{4}, \quad \operatorname{rank} K_{\rho}=2 .
$$

Conjugation by the unitary permutation matrix corresponding to the permutation (1)(24)(3) brings (3.4) to the form

$$
\left[\begin{array}{cc|cc}
\frac{a}{2} & z & y & x \\
\bar{z} & \frac{a}{2} & -\bar{x} & -\bar{y} \\
\hline \bar{y} & -x & \frac{1-a}{2} & \bar{t} \\
\bar{x} & -y & t & \frac{1-a}{2}
\end{array}\right]
$$

with rank 2. By Proposition 2.1 this implies that

$$
\left[\begin{array}{cc}
\frac{1-a}{2} & \bar{t} \\
t & \frac{1-a}{2}
\end{array}\right]=A^{\dagger} K A
$$

where

$$
A=K^{-1}\left[\begin{array}{cc}
y & x \\
-\bar{x} & -\bar{y}
\end{array}\right], \quad K=\left[\begin{array}{cc}
\frac{a}{2} & z \\
\bar{z} & \frac{a}{2}
\end{array}\right] .
$$

Putting $x=\frac{\sqrt{a(1-a)}}{2} \mathrm{e}^{\mathrm{i} \theta}, y=\frac{\sqrt{a(1-a)}}{2} \mathrm{e}^{\mathrm{i} \varphi}$, substituting the expressions of (3.8) in (3.7) and equating the 11-entry of the matrices on both sides of (3.7) we get

$$
\left|\frac{a}{2}+z \mathrm{e}^{-\mathrm{i}(\theta+\varphi)}\right|^{2}=0
$$

and therefore $|z|^{2}=\frac{a^{2}}{4}$, a contradiction.

The case $|t|^{2}<\frac{(1-a)^{2}}{4}$ is dealt with in the same manner.

Now we shall prove that $\rho$ is not extremal. Express (3.4) as

$$
K_{\rho}=\left[\begin{array}{c|c}
K & K A \\
\hline A^{\dagger} K & A^{\dagger} K A
\end{array}\right]
$$

where

$$
K=\left[\begin{array}{cc}
\frac{a}{2} & x \\
\bar{x} & \frac{1-a}{2}
\end{array}\right], \quad A=K^{-1}\left[\begin{array}{cc}
y & z \\
t & -y
\end{array}\right],
$$




$$
A^{\dagger} K A=d K^{-1}, \quad d=\frac{a(1-a)}{4}-|x|^{2}>0 .
$$

This implies the existence of a unitary matrix $U$ such that

$$
K^{1 / 2} A=d^{1 / 2} U K^{-1 / 2} .
$$

From (3.10) we have

$$
\left[\begin{array}{cc}
y & z \\
t & -y
\end{array}\right]=K A=d^{1 / 2} K^{1 / 2} U K^{-1 / 2} .
$$

Hence $\operatorname{Tr} U=0$. Since $U$ is a unitary matrix of zero trace it has the form

$$
U=\mathrm{e}^{\mathrm{i} \theta} V
$$

where $V$ is a selfadjoint unitary matrix of determinant -1 . In particular

$$
A=d^{1 / 2} \mathrm{e}^{\mathrm{i} \theta} K^{-1 / 2} V K^{-1 / 2}
$$

where $V$ is selfadjoint and unitary. We now examine the linear space

$$
\mathcal{D}=\left\{\left[I_{2} \mid A\right]\left(X_{1} \otimes I_{2}+I_{2} \otimes X_{2}\right)\left[\frac{I_{2}}{A^{\dagger}}\right] \mid X_{i} \text { is Hermitian for each } i\right\} .
$$

In the ordered basis $\left\{\boldsymbol{e}_{j}, j=1,2,3,4\right\}$ it is easily verified that $X_{1} \otimes I_{2}+I_{1} \otimes X_{2}$ in $\mathcal{D}$ varies over all matrices of the form

$$
\left\{\left[\begin{array}{c|c}
X+p I_{2} & r I_{2} \\
\hline \bar{r} I_{2} & X+q I_{2}
\end{array}\right] \mid X \text { Hermitian, } p, q \in \mathbb{R}, r \in \mathbb{C}\right\} .
$$

Thus

$$
\mathcal{D}=\left\{X+A X A^{\dagger}+r A^{\dagger}+\bar{r} A+q A A^{\dagger}+p I \mid X \text { Hermitian, } p, q \varepsilon \mathbb{R}, r \in \mathbb{C}\right\} .
$$

We now search for a Hermitian matrix $L$ of order 2 in $\mathcal{D}^{\perp}$ with respect to the scalar product $\left\langle X_{1} \mid X_{2}\right\rangle=\operatorname{Tr} X_{1} X_{2}$ for any two Hermitian matrices of order 2. In other words we search for a Hermitian $L$ satisfying

$$
\left.\begin{array}{l}
\operatorname{Tr} L=0, \quad \operatorname{Tr} L K^{-1 / 2} V K^{1 / 2}=0, \\
\operatorname{Tr} L\left(X+d K^{-1 / 2} V K^{-1 / 2} X K^{-1 / 2} V K^{-1 / 2}\right)=0
\end{array}\right\}
$$

for all Hermitian $X$. (Here we have substituted for $A$ from (3.12).)

Note that $\sqrt{d} K^{-1 / 2} V K^{-1 / 2}=B$ is a Hermitian matrix of determinant -1 . Thus (3.14) reduces to

$$
\operatorname{Tr} L=0, \quad \operatorname{Tr} L B=0, \quad L+B L B=0 .
$$

The matrix $B$ can be expressed as

$$
B=W D W^{\dagger}
$$

where $W$ is unitary and

$$
D=\left[\begin{array}{cc}
\alpha & 0 \\
0 & -\alpha^{-1}
\end{array}\right], \quad \alpha>0 .
$$

Then for any $\xi \in \mathbb{C}$ the Hermitian matrix

$$
L=W^{\dagger}\left[\begin{array}{ll}
0 & \xi \\
\bar{\xi} & 0
\end{array}\right] W
$$

satisfies (3.15). In other words $\mathcal{D}^{\perp} \neq\{0\}$ and therefore the linear space $\mathcal{D}$ in (3.13) is not the space of all Hermitian matrices of order 2 . Hence by Theorem 2.5, the state $\rho$ is not extremal. 
Thus every extremal state $\rho$ in $\mathcal{C}\left(\frac{1}{2} I, \frac{1}{2} I\right)$ is of rank 1 . Such an extremal state $\rho$ has the form

$$
\rho=|\Omega\rangle\langle\Omega|
$$

where

$$
\begin{aligned}
& |\Omega\rangle=\sum_{x, y \in\{0,1\}} a_{x y}|x y\rangle, \\
& \sum_{x, y}\left|a_{x y}\right|^{2}=1 .
\end{aligned}
$$

The fact that $|\Omega\rangle\langle\Omega|$ has its marginal operators equal to $\frac{1}{2} I$ implies that $\left(\left(a_{x y}\right)\right)=\frac{1}{\sqrt{2}}\left(\left(u_{x y}\right)\right)$ where $\left(\left(u_{x y}\right)\right)$ is a unitary matrix of order 2 . Putting

$$
\sum_{y=0}^{1} u_{x y}|y\rangle=\left|\psi_{x}\right\rangle
$$

we see that

$$
|\Omega\rangle=\frac{1}{\sqrt{2}}\left(|0\rangle\left|\psi_{0}\right\rangle+|1\rangle\left|\psi_{1}\right\rangle\right)
$$

where $\{|0\rangle,|1\rangle\}$ is the canonical orthonormal basis in $\mathbb{C}^{2}$ and $\left\{\left|\psi_{0}\right\rangle,\left|\psi_{1}\right\rangle\right\}$ is another orthonormal basis in $\mathbb{C}^{2}$ (which may coincide with $\{|0\rangle,|1\rangle\})$. Varying the orthonormal basis $\left\{\left|\psi_{0}\right\rangle,\left|\psi_{1}\right\rangle\right\}$ of $\mathbb{C}^{2}$ in (3.16) we get all the extremal states of $\mathcal{C}\left(\frac{1}{2} I, \frac{1}{2} I\right)$ as $|\Omega\rangle\langle\Omega|$.

\section{An example of a mixed extremal state in $\mathcal{C}\left(\frac{1}{n} I_{n}, \frac{1}{n^{2}} I_{n^{2}}\right)$ which is also nonseparable}

Let $A$ be a finite additive Abelian group of cardinality $n$, addition operation + and null element 0 . Choose and fix a symmetric bicharacter $\langle\cdot, \cdot\rangle$ on $A \times A$ satisfying

$$
\begin{aligned}
& \langle a, b\rangle=\langle b, a\rangle, \quad|\langle a, b\rangle|=1, \\
& \langle a, b+c\rangle=\langle a, b\rangle\langle a, c\rangle
\end{aligned}
$$

for all $a, b, c \in A$. Denote by $\mathcal{H}$ the Hilbert space $L^{2}(A)$ with respect to the counting measure in $A$ and consider the orthonormal basis:

$$
|a\rangle=1_{\{a\}}, \quad a \in A,
$$

where the right-hand side denotes the indicator function of the singleton $\{a\}$ in $A$. Define the unitary operators $U_{a}$, $V_{b}$ in $\mathcal{H}$ by

$$
\begin{aligned}
& U_{a}|c\rangle=|a+c\rangle, \\
& V_{b}|c\rangle=\langle b, c\rangle|c\rangle
\end{aligned}
$$

for all $a, b, c$ in $A$. Then we have the Weyl commutation relations

$$
U_{a} U_{b}=U_{a+b}, \quad V_{a} V_{b}=V_{a+b}, \quad V_{b} U_{a}=\langle a, b\rangle U_{a} V_{b} \quad \text { for all } a, b \in A .
$$

Put

$$
W_{x}=U_{a} V_{b}, \quad x=(a, b) \in A \times A .
$$


Then the family $\left\{W_{x}\right\}$ is irreducible and

$$
\operatorname{Tr} W_{x}^{\dagger} W_{y}=n \delta_{x y} .
$$

In particular $\left\{\frac{1}{\sqrt{n}} W_{x}, x \in A \times A\right\}$ is an orthonormal basis in the Hilbert space $\mathcal{B}(\mathcal{H})$ of all operators on $\mathcal{H}$ with the scalar product

$$
\langle X \mid Y\rangle=\operatorname{Tr} X^{\dagger} Y, \quad X, Y \in \mathcal{B}(\mathcal{H}) .
$$

Define the operator matrix

$$
P=\frac{1}{n^{2}}\left[W_{x}^{\dagger} W_{y}\right], \quad x, y \in A \times A,
$$

of order $n^{2}$ with entries from $\mathcal{B}(\mathcal{H})$. Then $P=P^{\dagger}=P^{2}$ and $\operatorname{Tr} P=n$, when $P$ is considered as an operator in $\mathcal{H} \otimes \mathcal{K}$ where $\mathcal{K}=L^{2}(A \times A)$. Thus $P$ is a projection of rank $n$ in an $n^{3}$-dimensional Hilbert space. Define the state

$$
\rho_{0}=\frac{1}{n} P
$$

Theorem 4.1. $\rho_{0}$ is an extremal state in the convex $\operatorname{set} \mathcal{C}\left(\frac{1}{n} I_{\mathcal{H}}, \frac{1}{n^{2}} I_{\mathcal{K}}\right)$ where $I_{\mathcal{H}}$ and $I_{\mathcal{K}}$ are the identity operators in $\mathcal{H}$ and $\mathcal{K}$ respectively. Furthermore, in the range of $\rho_{0}$ there does not exist a nonzero product vector of the form $u \otimes f, u \in \mathcal{H}, f \in \mathcal{K}$.

Proof. Observe that $\rho_{0}$ can be expressed in the block form

$$
\rho_{0}=\frac{1}{n^{3}}\left[\begin{array}{c|c}
I_{\mathcal{H}} & B \\
\hline B^{\dagger} & B^{\dagger} B
\end{array}\right]
$$

where $B=\left[W_{x}, x \in A \times A, x \neq 0\right]$ and $\operatorname{rank} \rho_{0}=\operatorname{rank} I_{\mathcal{H}}=n$. Now consider a Hermitian operator $L$ in $\mathcal{H}$ and put

$$
\alpha_{L}=\left[\begin{array}{c|c}
L & L B \\
\hline B^{\dagger} L & B^{\dagger} L B
\end{array}\right]
$$

Suppose that the relative traces of $\alpha_{L}$ in $\mathcal{H}$ and $\mathcal{K}$ vanish. This would, in particular, imply

$\operatorname{Tr} L W_{x}=0$ for all $x \in A \otimes A$.

Since the family $\left\{\frac{1}{\sqrt{n}} W_{x}, x \in A \times A\right\}$ is an orthonormal basis in $\mathcal{B}(\mathcal{H})$ it follows that $L=0$. In other words $\rho_{0}$ satisfies the conditions of Proposition 2.3 and therefore $\rho_{0}$ is an extreme point of the convex set $\mathcal{C}\left(\frac{1}{n} I_{\mathcal{H}}, \frac{1}{n^{2}} I_{\mathcal{K}}\right)$.

To prove the second part, suppose that there exists a nonzero product vector $u \otimes f$ in the range of $\rho_{0}$. It follows from (4.1) and (4.2) that

$$
P u \otimes f=u \otimes f
$$

or equivalently

$$
\frac{1}{n^{2}} \sum_{y \in A \times A} f(y) W_{y} u=f(x) W_{x} u \quad \text { for all } x \in A \times A .
$$

Thus the right-hand side is independent of $x$ and therefore

$$
f(x) W_{x} u=f(0,0) u .
$$

Since $u \otimes f \neq 0$ it follows that $f(0,0) \neq 0$ and therefore $f(x) \neq 0$ for every $x \in A \times A$. Thus $\mathbb{C} u$ is a 1-dimensional invariant subspace for the irreducible family $\left\{W_{x}, x \in A \times A\right\}$. This is a contradiction. 
Remark. The last part of Theorem 4.1 implies that the state $\rho_{0}$ is not separable in the sense that $\rho_{0}$ cannot be expressed as $\sum_{i} p_{i} \alpha_{i} \otimes \beta_{i}$, where $i$ runs over a finite index set $S,\left\{p_{i}\right\}$ is a probability distribution on $S,\left\{\alpha_{i}\right\}$ and $\left\{\beta_{i}\right\}$ are families of states in $\mathcal{H}$ and $\mathcal{K}$ respectively (see [5]).

Theorem 4.2. Let $\mathcal{H}, \mathcal{K}$ be Hilbert spaces of dimension $m, n$ respectively and let $\rho$ be a state in $\mathcal{H} \otimes \mathcal{K}$ such that $\rho \in \mathcal{C}\left(\frac{1}{m} I_{\mathcal{H}}, \frac{1}{n} I_{\mathcal{K}}\right)$. Then

$$
S(\rho) \geqslant\left|\log _{2} m-\log _{2} n\right|
$$

where $S(\rho)$ denotes the von Neumann entropy of $\rho$. In particular,

$$
\operatorname{rank} \rho \geqslant \frac{\max (m, n)}{\min (m, n)} .
$$

Proof. Consider a spectral decomposition of $\rho$ in the form

$$
\rho=\sum_{j=1}^{k} p_{j}\left|\Omega_{j}\right\rangle\left\langle\Omega_{j}\right|
$$

where $\left\{\left|\Omega_{j}\right\rangle, 1 \leqslant j \leqslant k\right\}$ is an orthonormal set and $\left\{p_{j}, 1 \leqslant j \leqslant k\right\}$ is a probability distribution with $p_{j}>0$ for every $j$. In particular, $\operatorname{rank}(\rho)=k$. Let $\left\{\left|e_{r}\right\rangle, 1 \leqslant r \leqslant m\right\},\left\{\left|f_{s}\right\rangle, 1 \leqslant s \leqslant n\right\}$ be orthonormal bases in $\mathcal{H}, \mathcal{K}$ respectively. Define

$$
P(j, r, s)=p_{j}\left|\left\langle e_{r} \otimes f_{s} \mid \Omega_{j}\right\rangle\right|^{2} .
$$

Then $P(\cdot, \cdot, \cdot)$ can be viewed as a joint probability distribution of three random variables $X, Y, Z$ assuming values in the sets $\{1,2, \ldots, k\},\{1,2, \ldots, m\},\{1,2, \ldots, n\}$ respectively. Using the symbol $H$ for the Shannon entropy as well as conditional entropy for random variables assuming a finite number of values we have

$$
H(X Y Z)=H(Y)+H(X Z \mid Y)=H(Z)+H(X Y \mid Z) .
$$

By the hypothesis on $\rho$ we conclude that $Y$ and $Z$ are uniformly distributed in $\{1,2, \ldots, m\}$ and $\{1,2, \ldots, n\}$ respectively. Thus we get

$$
\begin{aligned}
\log _{2} m-\log _{2} n & =H(Y)-H(Z)=H(X Y \mid Z)-H(X Z \mid Y) \\
& \leqslant H(X Y \mid Z) \leqslant H(X \mid Z) \leqslant H(X)=S(\rho) .
\end{aligned}
$$

Interchanging $Y$ and $Z$ in this argument and combining the two inequalities we get

$$
S(\rho) \geqslant\left|\log _{2} m-\log _{2} n\right| \text {. }
$$

This completes the proof of the first part. We have

$$
S(\rho)=-\sum_{j=1}^{k} p_{j} \log _{2} p_{j} \leqslant \log _{2} k
$$

which yields the second part.

Remark. It is interesting to note that, in view of Theorem 4.2, the extremal state $\rho_{0}$ constructed in Theorem 4.1 is, indeed, of minimal rank.

We conclude with an example which is of some interest, particularly, in the context of Theorems 3.1 and 4.1 with $n=2$ which cover the cases $\mathbb{C}^{2} \otimes \mathbb{C}^{2}$ and $\mathbb{C}^{2} \otimes \mathbb{C}^{4}$. 
Example 4.3. Let $\mathcal{H}=\mathbb{C}^{2}, \mathcal{K}=\mathbb{C}^{3}$ with labeled orthonormal bases $\{|0\rangle,|1\rangle\},\{|0\rangle,|1\rangle,|2\rangle\}$ respectively. Suppose $\rho_{0}=\frac{1}{2} P$ where $P$ is the 2 -dimensional projection in $\mathcal{H} \otimes \mathcal{K}$ onto the span of $\{|00\rangle+|11\rangle+\mathrm{i}|12\rangle,|10\rangle+|01\rangle-$ $\mathrm{i}|02\rangle\}$. Using the ordered orthonormal basis $\{|00\rangle,|10\rangle,|01\rangle,|11\rangle,|02\rangle,|12\rangle\}$ in $\mathcal{H} \otimes \mathcal{K}$ and looking upon $\mathcal{H} \otimes \mathcal{K}$ as $\mathbb{C}^{2} \oplus \mathbb{C}^{2} \oplus \mathbb{C}^{2}, P$ can be expressed as a block matrix:

$$
P=\frac{1}{3}\left[\begin{array}{c|c|c}
I_{2} & \sigma_{1} & \sigma_{2} \\
\hline \sigma_{1} & I_{2} & \mathrm{i} \sigma_{3} \\
\hline \sigma_{2} & -\mathrm{i} \sigma_{3} & I_{2}
\end{array}\right]
$$

where $\sigma_{i}, i=1,2,3$, are the $2 \times 2$ Pauli matrices. Since the trace of any Pauli matrix is 0 it follows that $\rho_{0} \in$ $\mathcal{C}\left(\frac{1}{2} I_{2}, \frac{1}{3} I_{3}\right)$. It is straightforward to verify that there is no product vector in the range of $P$. Thus $\rho_{0}$ is a mixed entangled state with both the marginals having maximum entropy. If $L$ is a $2 \times 2$ Hermitian matrix such that the marginals of the operator

$$
T_{L}=\left[\begin{array}{c|c|c}
L & L \sigma_{1} & L \sigma_{2} \\
\hline \sigma_{1} L & \sigma_{1} L \sigma_{1} & \sigma_{1} L \sigma_{2} \\
\hline \sigma_{2} L & \sigma_{2} L \sigma_{1} & \sigma_{2} L \sigma_{2}
\end{array}\right]
$$

in $\mathcal{H}$ and $\mathcal{K}$ are 0 then it follows that $\operatorname{Tr} L=\operatorname{Tr} L \sigma_{1}=\operatorname{Tr} L \sigma_{2}=\operatorname{Tr} L \sigma_{3}=0$ and therefore $L=0$. By Proposition 2.4 it follows that $\rho_{0}$ is an extremal state in $\mathcal{C}\left(\frac{1}{2} I_{2}, \frac{1}{3} I_{3}\right)$. By Theorem 4.2, $\rho_{0}$ has minimal rank.

\section{Acknowledgements}

The author is grateful to the anonymous referee at whose suggestion Theorem 4.2, the subsequent interesting remark and Example 4.3 with some modifications have been added.

\section{References}

[1] R.B. Bapat, T.E.S. Raghavan, Nonnegative Matrices and Applications, Encyclopaedia of Mathematics and its Applications, vol. 64, Cambridge University Press, 1997.

[2] G. Birkhoff, Three observations on linear algebra, Univ. Nac. Tucumán Rev. Ser. A 5 (1946) 147-151.

[3] J.P.R. Christensen, J. Vesterstrom, A note on extreme positive definite matrices, Math. Ann. 244 (1979) 65-68.

[4] R. Grome, S. Pierce, W. Watkins, Extremal correlation matrices, Linear Algebra Appl. 134 (1990) 63-70.

[5] M. Horodecki, P. Horodecki, R. Horodecki, Separability of mixed states: necessary and sufficient conditions, Phys. Lett. A 223 (1-2) (1996) $1-8$.

[6] R. Loewy, Extreme points of a convex subset of the cone of positive semidefinite matrices, Math. Ann. 253 (1980) $227-232$.

[7] K.R. Parthasarathy, On extremal correlations, J. Statist Plann. Inference 103 (2002) 73-80. 